\title{
New Versatile Precursors of Functional RAFT agents. Application to the Synthesis of Bio-Related End-Functionalized Polymers.
}

\author{
Maël Bathfield ${ }^{1,2}$, Franck D'Agosto ${ }^{2}$, Roger Spitz ${ }^{2}$, Marie-Thérèse Charreyre ${ }^{1}$, Thierry Delair $^{1}$ \\ Contribution from 1) the Unité Mixte CNRS-bioMérieux, École Normale Supérieure de Lyon, 46 allée d'Italie, 69364 \\ Lyon Cedex 07, France and 2) the Laboratoire de Chimie et Procédés de Polymérisation UMR 140 CNRS/ESCPE, \\ Bât 308 F, 43 Boulevard du 11 Novembre 1918, BP 2077, 69616 Villeurbanne CEDEX, France
}

\section{Materials}

N-Acryloylmorpholine (NAM) (Aldrich, 97\%) was distilled under reduced pressure $\left(120{ }^{\circ} \mathrm{C}\right.$, 10 mmHg). 4,4'-Azobis(isobutyronitrile) (AIBN) (Fluka, 98\%) was purified by recrystallization from ethanol. 1,4-Dioxane (Acros, 99\%) was distilled over $\mathrm{LiAlH}_{4}\left(110^{\circ} \mathrm{C}\right)$. Tetrahydrofuran (Aldrich, $>99.5 \%$ ) was distilled over $\mathrm{Na}$ (in the presence of benzophenone). Phenylmagnesium bromide solution (1.0 $\mathrm{M}$ in tetrahydrofuran, Aldrich), carbon disulfide (Aldrich, >99\%), $( \pm$ )-2-Bromopropionic acid (Aldrich, $>99 \%$ ), $N$ Hydroxysuccinimide (Aldrich, 98\%), N,N'-Dicyclohexylcarbodiimide (Aldrich, 99\%), 4-(2aminoethyl)morpholine (Aldrich, 99\%), EZ-Link ${ }^{\mathrm{TM}}$ Biotin PEO-Amine (Pierce), 1,2:3,4-Di$O$-isopropylidene- $\alpha$-D-galactopyranose (Aldrich, 97\%), trioxane (Acros, 99\%), and other materials were all used without further purification.

\section{Analytical techniques}

Both ${ }^{1} \mathrm{H}$ NMR $(200 \mathrm{MHz})$ and ${ }^{13} \mathrm{C}$ NMR $(50 \mathrm{MHz})$ spectra were recorded on a Bruker AC 200 spectrometer at $27^{\circ} \mathrm{C}$ in $d$-chloroform $\left(\mathrm{CDCl}_{3}\right)$.

Molecular weight distributions were determined by size exclusion chromatography (SEC) coupled to a light scattering detection (LSD). Aqueous SEC was performed using a Waters 510 pump and two Waters Ultrahydrogel columns (2000 and $500 \AA$ ). and. On-line double detection was provided by a differential refractometer (DRI Waters 410) and a three-angle $\left(47^{\circ}, 90^{\circ}, 130^{\circ}\right)$ MiniDAWN light scattering photometer (Wyatt Technologies), operating at $690 \mathrm{~nm}$. Analyses were performed by injection of $200 \mu \mathrm{L}$ of polymer solution $\left(5 \mathrm{mg} \cdot \mathrm{mL}^{-1}\right)$ in a borate buffer ( $\left.\mathrm{pH}=9.3,0.05 \mathrm{~mol} . \mathrm{L}^{-1}\right)$, previously filtered through a $0.22 \mu \mathrm{m}$ Millipore filter and used as eluent at a flow rate of $0.5 \mathrm{~mL} \min ^{-1}\left(35^{\circ} \mathrm{C}\right)$. The specific refractive index increment $(\mathrm{dn} / \mathrm{dc})$ for poly(NAM) in the same eluent $(0.163)$ was previously determined with a NFT ScanRef monocolor interferometer operating at $633 \mathrm{~nm}$. The molecular weight and polydispersity data were determined using the Wyatt ASTRA SEC/LS software package.

The matrix-assisted laser desorption ionization time-of-flight mass spectrometer (MALDIToF MS) was a Voyager-DE STR (Applied Biosystems, Foster City, CA). This instrument was equipped with a nitrogen laser (wavelength $337 \mathrm{~nm}$ ). The accelerating voltage was 20 $\mathrm{kV}$. The positive ions were detected in all cases. The spectra were the sum of 200 shots, and an external mass calibration was used (mixture of peptides standards, Sequazyme kit).The samples were deposited on a stainless steel target and dried. Samples were prepared by dissolving the polymer in freshly distilled tetrahydrofuran (THF) at a concentration of 10 g. $\mathrm{L}^{-}$ ${ }^{1}$. The matrix was $\alpha$-cyano-4-hydroxycinnamic acid (CHCA, Sigma-Aldrich), used without further purification and dissolved in distilled THF $\left(10\right.$ g.L $\left.{ }^{-1}\right)$. NaI solution (10 g. $\mathrm{L}^{-1}$ in acetone) was used to enhance sodium ionization. Matrix, NaI and polymer solutions were mixed at a volume ratio of 9:1:1 ; then $1 \mu \mathrm{L}$ of the mixture was deposited onto the MALDI target before insertion into the ion source chamber. 
Elemental analyses and mass spectrometry were carried out by the "Service Central d'Analyses du CNRS" (Solaize, France). The mass spectra were obtained on a ZAB2-SEQ spectrometer (VG, Manchester, UK) using fast atom bombardment ionization.

\section{Methods}

Polymerization procedure. NAM (1.5 g, $10.6 \mathrm{mmol})$, RAFT agent 3a (10.2 mg, $0.03 \mathrm{mmol})$, AIBN (0.49 mg, $0.003 \mathrm{mmol})$, dioxane $(5.31 \mathrm{~mL})$, and trioxane $(0.08 \mathrm{~g}$, internal reference for ${ }^{1} \mathrm{H}$ NMR determination of monomer consumption) were introduced in a Schlenk tube equipped with a magnetic stirrer. The mixture was degassed by five freeze-evacuate-thaw cycles and then heated under nitrogen in a thermostated oil bath. Periodically, samples were withdrawn from the polymerization medium for analyses.

Monomer conversion was determined by ${ }^{1} \mathrm{H}$ NMR spectroscopy using a Bruker AC 200 spectrometer (200 MHz), by comparison of one vinylic proton (5.7 ppm) with trioxane (5.1 ppm) used as internal reference ${ }^{1}$. Typically, $400 \mu \mathrm{L}$ of $d$-chloroform was added to $200 \mu \mathrm{L}$ of each sample.

Polymers were precipitated in a large volume of diethyl ether, recovered by centrifugation, and finally dried under air before analysis by size exclusion chromatography.

\section{Synthesis of succinimido-2-[[2-phenyl-1-thioxo]thio]-propanoate (precursor RAFT agent}

1).

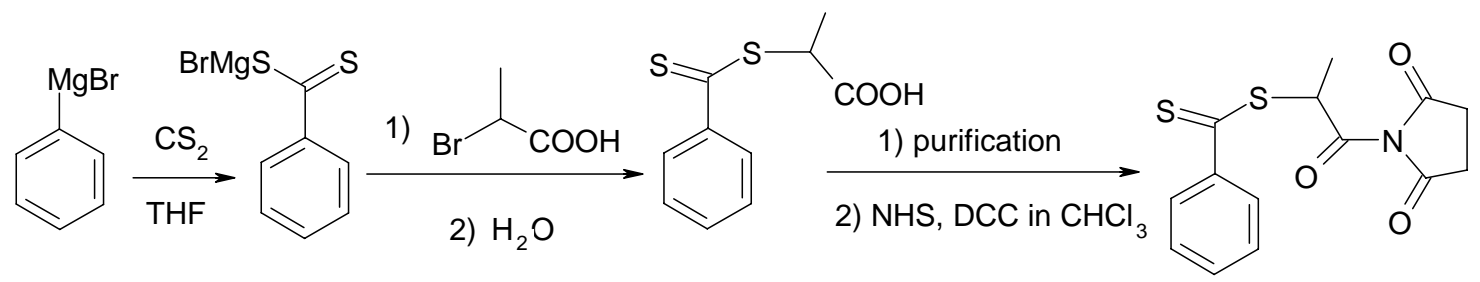

Scheme 1: Synthetic scheme for succinimido-2-[[2-phenyl-1-thioxo]thio]-propanoate

2-[[2-phenyl-1-thioxo]thio]-propanoic acid was synthesized according to the literature procedure. ${ }^{2}$ Purification by silica gel chromatography (Silica gel 60, Merk) with ethyl acetate/petroleum ether $(60 / 40: \mathrm{v} / \mathrm{v})$ as eluent was performed and a crystallized pure red product was obtained (yield 46\%).

In an argon conditioned Schlenk tube equipped with a magnetic stirrer, the freshly prepared dithioester (1.64 g, $7.2 \mathrm{mmol})$ was dissolved in chloroform $(100 \mathrm{~mL})$. After cooling to $0^{\circ} \mathrm{C}, \mathrm{N}$ Hydroxysuccinimide $(0.84 \mathrm{~g}, 7.2 \mathrm{mmol})$ and $N, N^{\prime}$-Dicyclohexylcarbodiimide $(1.50 \mathrm{~g}, 7.2$ mmol) were successively added. After $1 \mathrm{~h}$ at $0^{\circ} \mathrm{C}$ and $22 \mathrm{~h}$ at room temperature, the reaction mixture was filtrated. After solvent removal, the product was twice dissolved in a minimum volume of ethyl acetate and filtrated. After drying under vacuum, a very viscous orange-red oil was obtained (yield 96\%).

${ }^{1} \mathrm{H}$ NMR $200 \mathrm{MHz}\left(\mathrm{CDCl}_{3}, \mathrm{RT}\right.$, ppm) (see structure below for proton assignment): 1.82 (d, $\mathrm{H}_{5}$ ); 2.83 (s, $\mathrm{H}_{6}$ ); 5.09 (q, $\left.\mathrm{H}_{4}\right) ; 7.40$ (dd, $\mathrm{H}_{2}$ ); 7.56 (dd, $\mathrm{H}_{1}$ ); 8.01 (d, $\mathrm{H}_{3}$ ).<smiles>O=C(ON1C(=O)CCC1=O)C(S)SC(=S)c1ccccc1</smiles> 


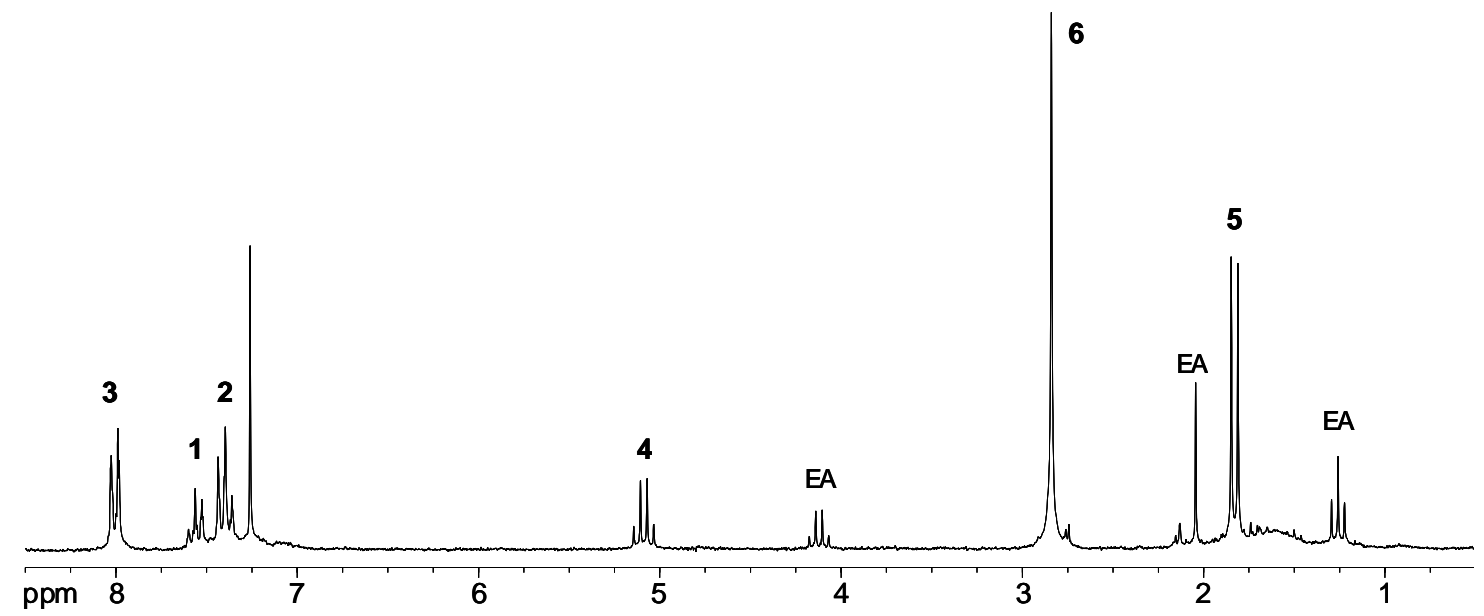

Figure 1: ${ }^{1} \mathrm{H}$ NMR spectrum of RAFT agent 1 in $\mathrm{CDCl}_{3}$; [EA = ethyl acetate]

${ }^{13} \mathrm{C}$ NMR 50MHz $\left(\mathrm{CDCl}_{3}, \mathrm{RT}\right.$, ppm) (see structure below for carbon assignment): $16.43\left(\mathrm{C}_{7}\right)$; $25.61\left(\mathrm{C}_{10}\right) ; 45.70\left(\mathrm{C}_{6}\right) ; 127.11\left(\mathrm{C}_{3}\right) ; 128.53\left(\mathrm{C}_{2}\right) ; 133.05\left(\mathrm{C}_{1}\right) ; 143.84\left(\mathrm{C}_{4}\right) ; 167.28\left(\mathrm{C}_{8}\right)$; $168.64\left(\mathrm{C}_{9}\right) ; 223.96\left(\mathrm{C}_{5}\right)$.
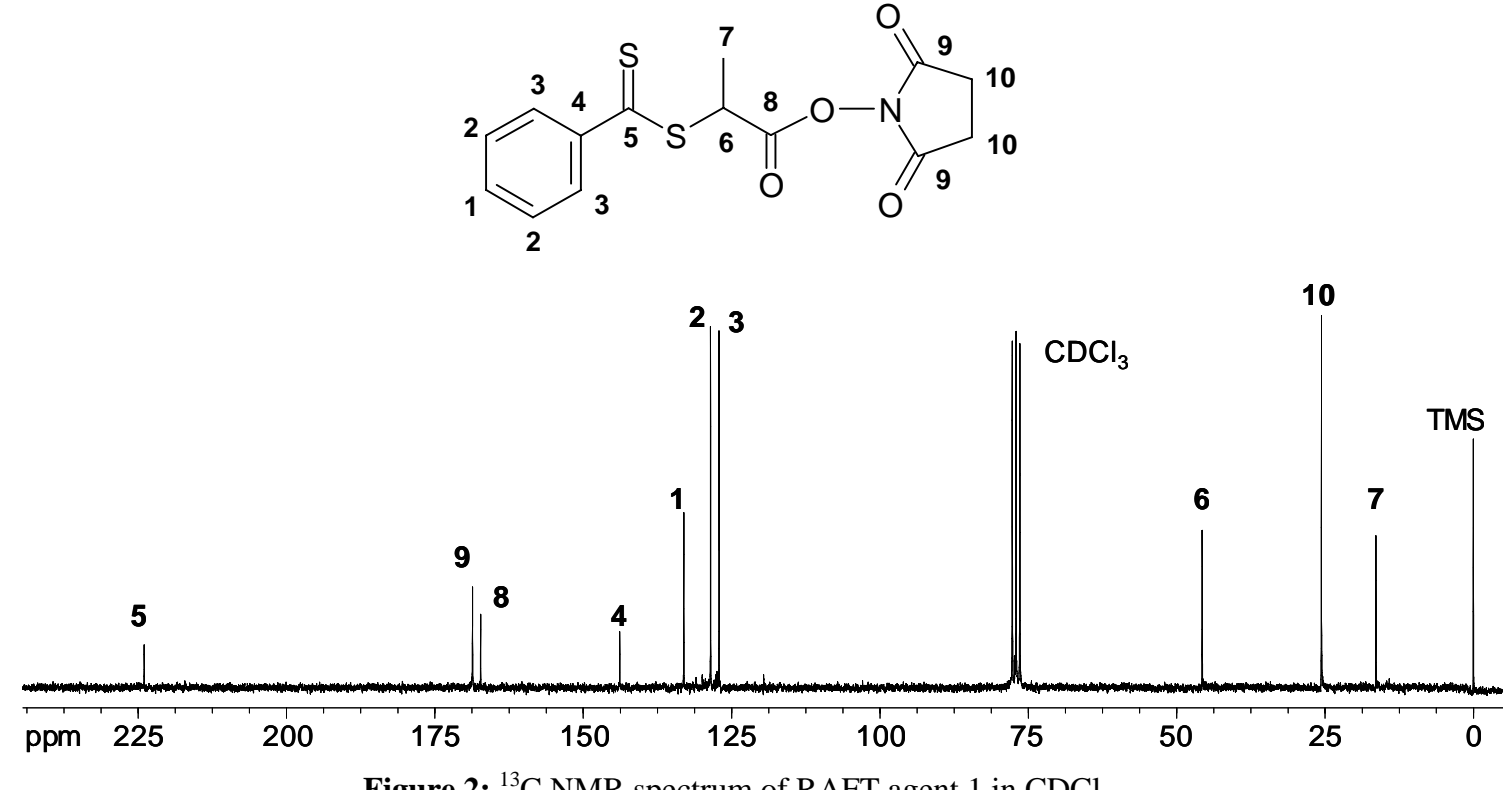

Figure 2: ${ }^{13} \mathrm{C}$ NMR spectrum of RAFT agent 1 in $\mathrm{CDCl}_{3}$.

Succinimido-2-[[2-phenyl-1-thioxo]thio]-propanoate (precursor RAFT agent 1) fragmentation undergoes during Fast Atom Bombardment (FAB) mass spectrometry.

It is used as obtained for the syntheses of the bio-related RAFT agents.

Anal. Calcd. for $\mathrm{C}_{14} \mathrm{H}_{13} \mathrm{NO}_{4} \mathrm{~S}_{2}$ : C, 52.00; H, 4.05; N, 4.33; S, 19.83. Found: C, 50.25; H, 4.15;

N, 4.07; S, 18.58

Weight purity $=95 \%$ 
Synthesis of N-(2-morpholinoethyl)-2-[[2-phenyl-1-thioxo]thio]-propanamide (RAFT agent 3a). In a $100 \mathrm{~mL}$ round-bottomed flask equipped with a magnetic stirrer, RAFT agent 1 ( $0.5 \mathrm{~g}$ of raw product corresponding to $1.47 \mathrm{mmol}$ of pure dithioester) was dissolved in chloroform $(60 \mathrm{~mL})$. A solution of 4-(2-aminoethyl)morpholine $(0.18 \mathrm{~g}, 1.39 \mathrm{mmol})$ in $10 \mathrm{~mL}$ of chloroform was added in one portion to the solution of RAFT agent $\mathbf{1}$. The reaction mixture was stirred at $30^{\circ} \mathrm{C}$ for $1 \mathrm{~h} 30$. Then, it was washed three times with $250 \mathrm{~mL}$ of distilled water (to remove NHS) and dried over anhydrous magnesium sulfate. After solvent removal, two successive column chromatography (Silica gel 60, Merk) were performed (dichloromethane/ethyl acetate: 50/50 v/v then acetone; dichloromethane/ethanol: $95 / 5 \mathrm{v} / \mathrm{v}$ ) to afford an orange-red oil (yield 68\%; for purity sake, all fractions containing traces of impurities (NHS and impurities from the precursor RAFT agent 1) were excluded).

${ }^{1} \mathrm{H}$ NMR $200 \mathrm{MHz}\left(\mathrm{CDCl}_{3}\right.$, RT, ppm) (see structure below for proton assignment): 1.68 (d, $\left.\mathrm{H}_{5}\right)$; 2.36-2.46 (m, $\mathrm{H}_{8}$ and $\left.\mathrm{H}_{9}\right) ; 3.33$ (dt, $\left.\mathrm{H}_{7}\right) ; 3.63$ (t, $\left.\mathrm{H}_{10}\right) ; 4.72$ (q, $\left.\mathrm{H}_{4}\right) ; 6.95\left(\mathrm{~s}, \mathrm{H}_{6}\right) ; 7.40$ (dd, $\left.\mathrm{H}_{2}\right) ; 7.56$ (dd, $\left.\mathrm{H}_{1}\right) ; 8.00$ (d, $\mathrm{H}_{3}$ ).
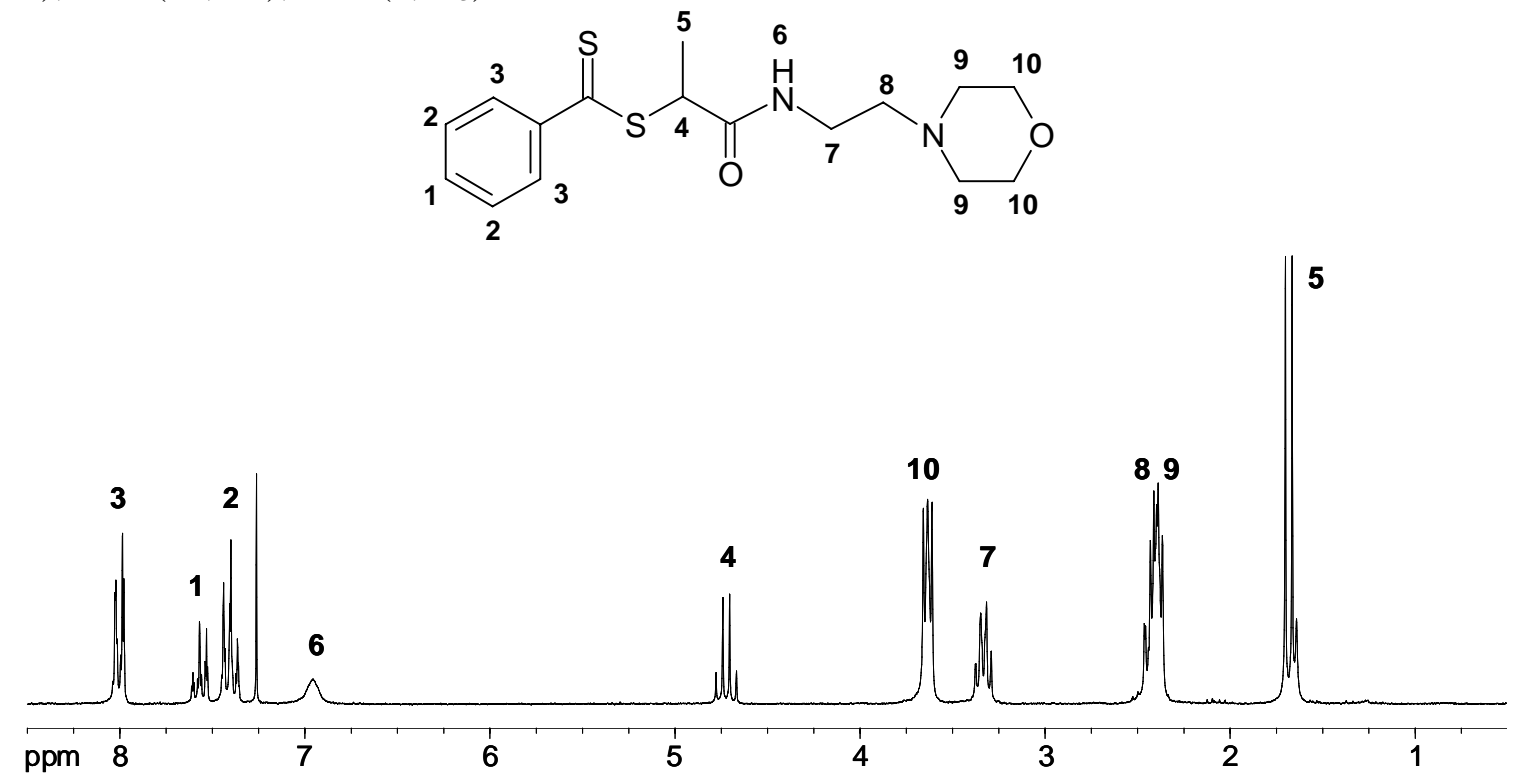

Figure 3: ${ }^{1} \mathrm{H}$ NMR spectrum of RAFT agent 3a in $\mathrm{CDCl}_{3}$.

${ }^{13} \mathrm{C}$ NMR 50MHz $\left(\mathrm{CDCl}_{3}, \mathrm{RT}\right.$, ppm) (see structure below for carbon assignment): $16.02\left(\mathrm{C}_{7}\right)$; $36.04\left(\mathrm{C}_{9}\right)$; $48.21\left(\mathrm{C}_{6}\right) ; 53.23\left(\mathrm{C}_{11}\right) ; 56.58\left(\mathrm{C}_{10}\right) ; 66.99\left(\mathrm{C}_{12}\right) ; 127.03\left(\mathrm{C}_{3}\right) ; 128.53\left(\mathrm{C}_{2}\right) ; 133.08$ $\left(\mathrm{C}_{1}\right) ; 144.19\left(\mathrm{C}_{4}\right) ; 170.31\left(\mathrm{C}_{8}\right) ; 227.15\left(\mathrm{C}_{5}\right)$<smiles>CC(SC(=S)c1ccccc1)C(=O)NCCN1CCOCC1</smiles> 


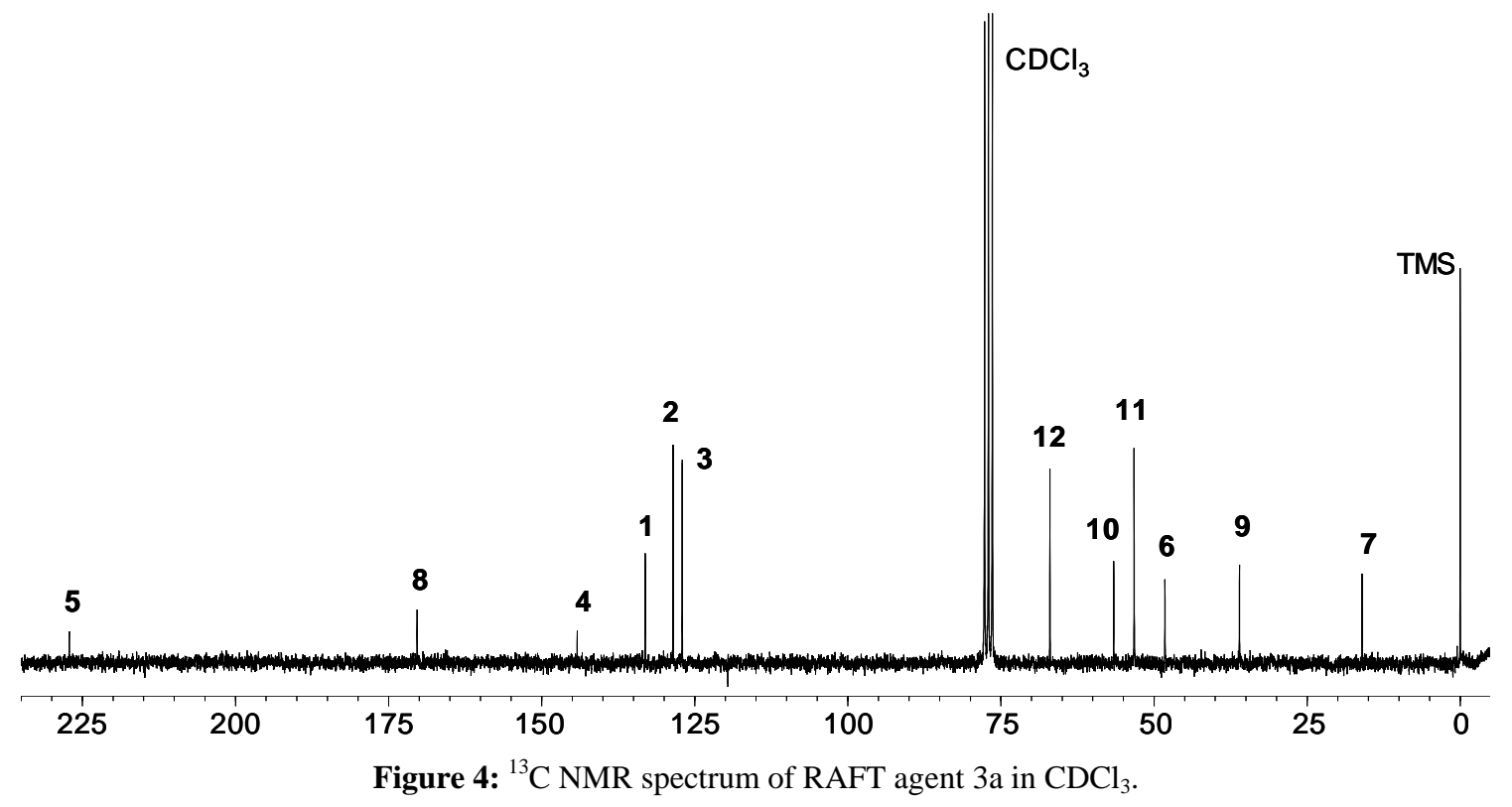

Fast Atom Bombardment (FAB) mass spectrometry: characteristic ion $\left[\mathrm{M}+\mathrm{H}^{+}\right]$: $\mathrm{C}_{16} \mathrm{H}_{23} \mathrm{~N}_{2} \mathrm{O}_{2} \mathrm{~S}_{2}$; calculated 339.1201; found 339.1205.

Anal. Calcd. for $\mathrm{C}_{16} \mathrm{H}_{22} \mathrm{~N}_{2} \mathrm{O}_{2} \mathrm{~S}_{2}$ : C, 56.77; H, 6.55; N, 8.28; S, 18.95. Found: C, 56.86; H, 6.57; N, 8.15; S, 19.02

Synthesis of $\mathrm{N}$-[6-desoxy-1,2:3,4-di-O-isopropylidene-6- $\alpha$-D-galactopyranosyl]-2-[[2phenyl-1-thioxoethyl]thio]-propanamide (RAFT agent 3b). 6-amino-6-desoxy-1,2:3,4-di$O$-isopropylidene-6- $\alpha$-D-galactopyranose (sugar- $\mathrm{NH}_{2}$ ) was synthesised from 1,2:3,4-Di-Oisopropylidene- $\alpha$-D-galactopyranose according to B. Badey et al. ${ }^{3}$. In a $50 \mathrm{~mL}$ roundbottomed flask equipped with a magnetic stirrer, RAFT agent 1 ( $0.28 \mathrm{~g}$ of raw product corresponding to $0.82 \mathrm{mmol}$ of pure dithioester) was dissolved in chloroform (30 mL). A solution of sugar- $\mathrm{NH}_{2}(0.2 \mathrm{~g}, 0.78 \mathrm{mmol})$ in $4 \mathrm{~mL}$ of chloroform was separately prepared. The latter was added in four fractions (about 15 min between each addition) to the solution of RAFT agent 1 . The reaction mixture was stirred at $30^{\circ} \mathrm{C}$ for $2 \mathrm{~h}$. Then, it was washed three times with $200 \mathrm{~mL}$ of distilled water and dried over anhydrous magnesium sulfate. After solvent removal, two successive column chromatography (Silica gel 60, Merk) were performed (dichloromethane/ethyl acetate/ethanol: 92/5/3 v/v/v; dichloromethane/ethyl acetate/ethanol: 79/20/1 v/v/v) to afford a very viscous red oil (yield 66\%; for purity sake, all fractions containing traces of impurities (NHS and impurities from the precursor RAFT agent 1) were excluded).

${ }^{1} \mathrm{H}$ NMR $200 \mathrm{MHz}\left(\mathrm{CDCl}_{3}\right.$, RT, ppm) (see structure below for proton assignment): 1.22, 1.31 and $1.44\left(\mathrm{~s}, \mathrm{H}_{13}\right) ; 1.66\left(\mathrm{~d}, \mathrm{H}_{5}\right)$; 3.13-3.34 (m, $\left.\mathrm{H}_{7}\right)$; 3.58-3.92 (m, $\mathrm{H}_{7}$, and $\left.\mathrm{H}_{8}\right)$; 4.10-4.17 (m, $\mathrm{H}_{11}$ ); 4.25-4.30 (m, $\left.\mathrm{H}_{9}\right)$; 4.54-4.59 (m, $\left.\mathrm{H}_{10}\right)$; 4.70 (q, $\left.\mathrm{H}_{4}\right)$; 5.45-5.51 (m, $\left.\mathrm{H}_{12}\right)$; 6.62-6.71 (d, $\mathrm{H}_{6}$ ); 7.38 (dd, $\mathrm{H}_{2}$ ); 7.54 (dd, $\left.\mathrm{H}_{1}\right) ; 7.99$ (m, $\mathrm{H}_{3}$ ).

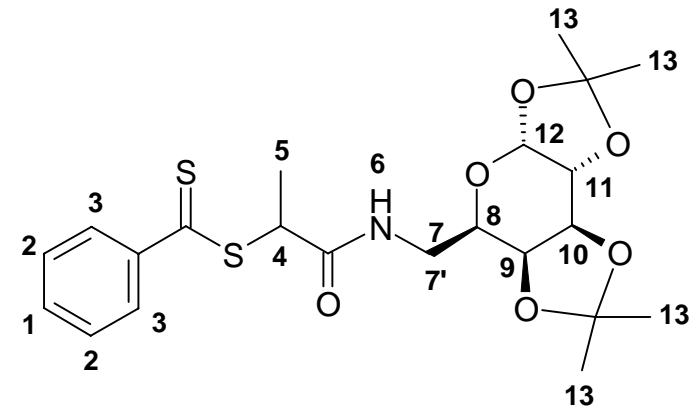




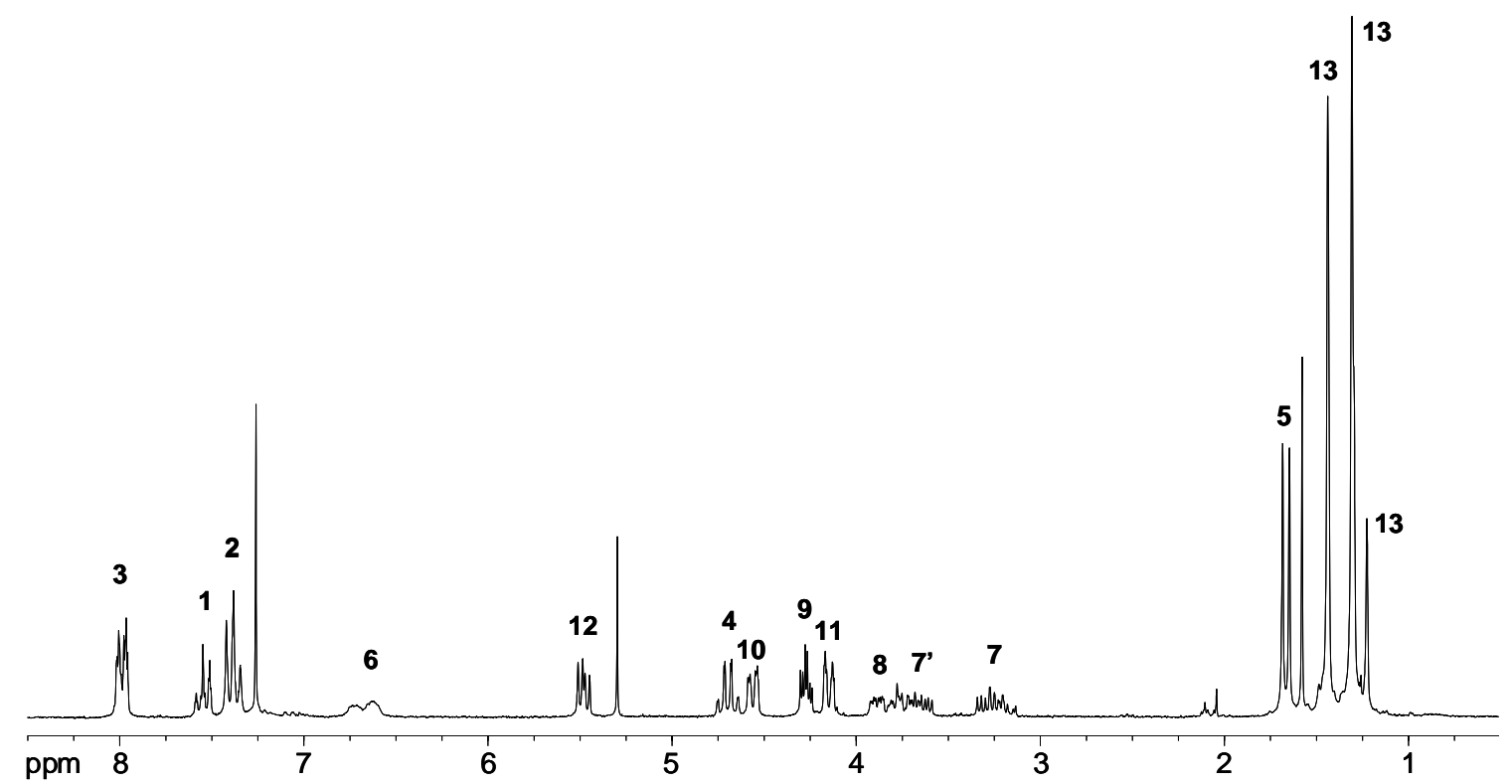

Figure 5: ${ }^{1} \mathrm{H}$ NMR spectrum of RAFT agent $3 \mathrm{~b}$ in $\mathrm{CDCl}_{3}$.

${ }^{13} \mathrm{C}$ NMR $50 \mathrm{MHz}\left(\mathrm{CDCl}_{3}, \mathrm{RT}\right.$, ppm) (see structure below for carbon assignment): 16.15, $16.47\left(\mathrm{C}_{7}\right)$; 24.28, 24.97, $25.97\left(\mathrm{C}_{16}\right)$; 40.12, $40.24\left(\mathrm{C}_{9}\right)$; 48.21, $48.77\left(\mathrm{C}_{6}\right)$; 66.06, 66.33, $67.10\left(\mathrm{C}_{11}, \mathrm{C}_{12}, \mathrm{C}_{13}\right)$; 70.54, 70.80, $71.58\left(\mathrm{C}_{10}\right)$; $96.24\left(\mathrm{C}_{14}\right)$; 108.70, $109.44\left(\mathrm{C}_{15}\right)$; 127.09, $127.24\left(\mathrm{C}_{3}\right)$; 128.30, $128.40\left(\mathrm{C}_{2}\right)$; 132.76, $132.83\left(\mathrm{C}_{1}\right)$; $144.46\left(\mathrm{C}_{4}\right) ; 170.61,170.66\left(\mathrm{C}_{8}\right)$; 226.84, $227.04\left(\mathrm{C}_{5}\right)$.
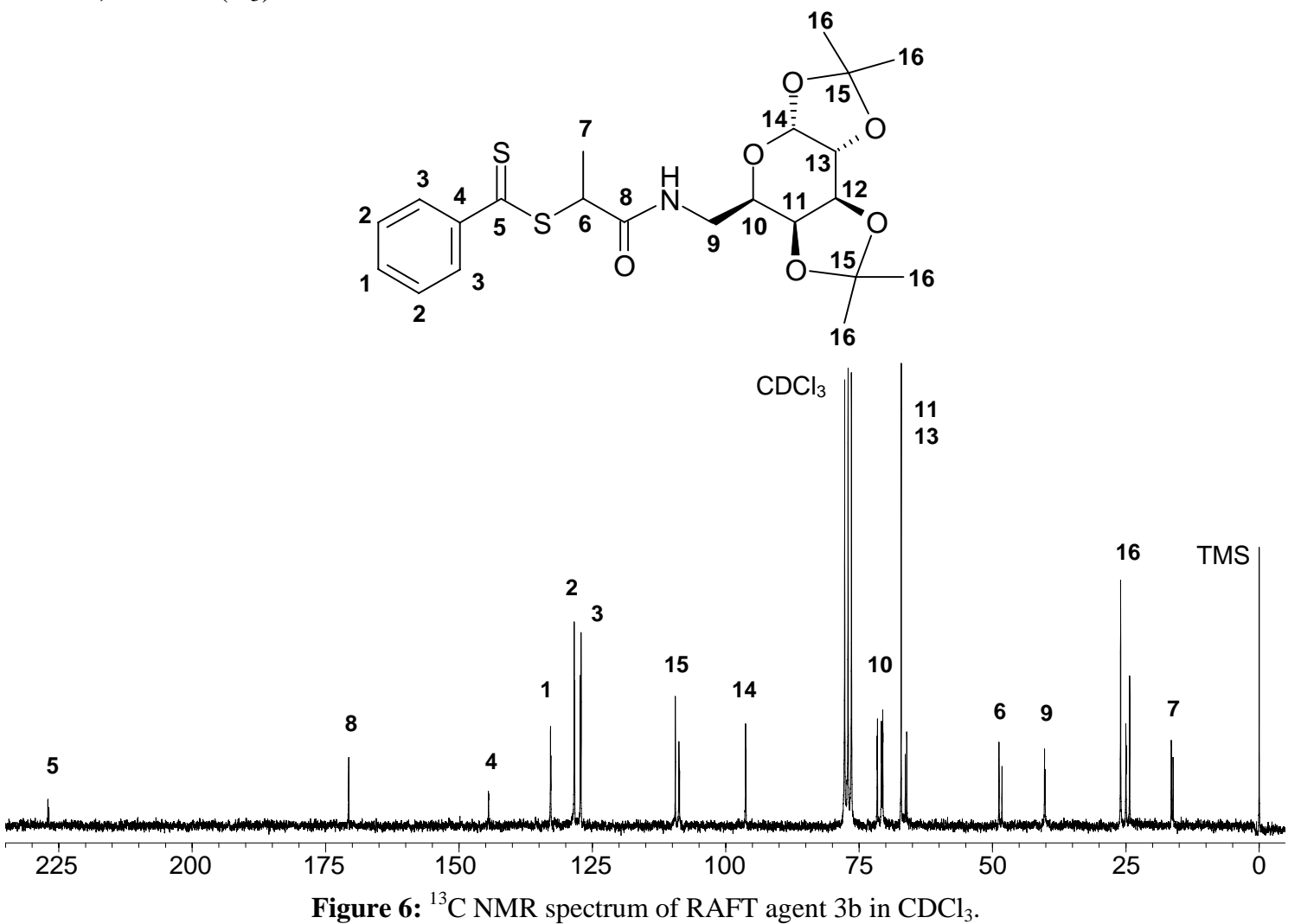

Fast Atom Bombardment (FAB) mass spectrometry: characteristic ion $\left[\mathrm{M}+\mathrm{H}^{+}\right]$: $\mathrm{C}_{22} \mathrm{H}_{30} \mathrm{NO}_{6} \mathrm{~S}_{2}$; calculated 468.1515; found 468.1532.

Anal. Calcd. for $\mathrm{C}_{22} \mathrm{H}_{29} \mathrm{NO}_{6} \mathrm{~S}_{2}$ : C, 56.51; H, 6.25; N, 3.00; S, 13.71. Found: C, 54.27; H, 6.01; $\mathrm{N}, 2.76 ; \mathrm{S}, 13.11$ 
Synthesis of N-[8-((+)-biotinamido)-3,6-dioxaoctyl]-2-[[2-phenyl-1-thioxoethyl]thio]propanamide (RAFT agent 3c). In a $100 \mathrm{~mL}$ round-bottomed flask equipped with a magnetic stirrer, RAFT agent 1 (0.18 g of raw product corresponding to $0.53 \mathrm{mmol}$ of pure dithioester) was dissolved in chloroform $(20 \mathrm{~mL})$. A solution of EZ-Link ${ }^{\mathrm{TM}}$ Biotin PEOAmine ((+)-Biotinyl-3, 6-dioxaoctanediamine, $0.19 \mathrm{~g}, 0.50 \mathrm{mmol})$ in $30 \mathrm{~mL}$ of chloroform was separately prepared. The latter was added in three fractions (about 20 min between each addition) to the solution of RAFT agent 1 . The reaction mixture was stirred at $30^{\circ} \mathrm{C}$ for $2 \mathrm{~h}$. Then, it was washed three times with $200 \mathrm{~mL}$ of distilled water and dried over anhydrous magnesium sulfate. After solvent removal, two successive column chromatographies (Silica gel 60, Merk) were performed (acetone then ethanol; acetone then dichloromethane/ethanol: $70 / 30 \mathrm{v} / \mathrm{v}$ ) to afford a very viscous red oil (yield 72\%; for purity sake, all fractions containing traces of impurities (NHS and impurities from the precursor RAFT agent 1) were excluded).

${ }^{1} \mathrm{H}$ NMR $200 \mathrm{MHz}\left(\mathrm{CDCl}_{3}, \mathrm{RT}\right.$, ppm) (see structure below for proton assignment): 1.44 (dt, $\mathrm{H}_{17}$ ); 1.68 (d, $\mathrm{H}_{5}$ ); 1.68 (overlapping previous signal, $\mathrm{H}_{15}$ and $\mathrm{H}_{16}$ ); 2.21 (t, $\left.\mathrm{H}_{14}\right)$; 2.69-2.75 (d, $\mathrm{H}_{21}$ ); 2.85-2.94 (dd, $\mathrm{H}_{20}$ ); 3.12 (dt, $\mathrm{H}_{18}$ ); 3.40-3.57 (m, $\mathrm{H}_{7}$ to $\mathrm{H}_{12}$ ); 4.26-4.47 (m, $\mathrm{H}_{19}$ ); 4.68 (q, $\mathrm{H}_{4}$ ); 5.30 and 6.26 (s, $\mathrm{H}_{22}$ and $\left.\mathrm{H}_{23}\right) ; 6.48$ (s, $\mathrm{H}_{13}$ ); 7.01 (s, $\mathrm{H}_{6}$ ); 7.40 (dd, $\mathrm{H}_{2}$ ); 7.55 (dd, $\mathrm{H}_{1}$ ); $7.99\left(\mathrm{~d}, \mathrm{H}_{3}\right)$.
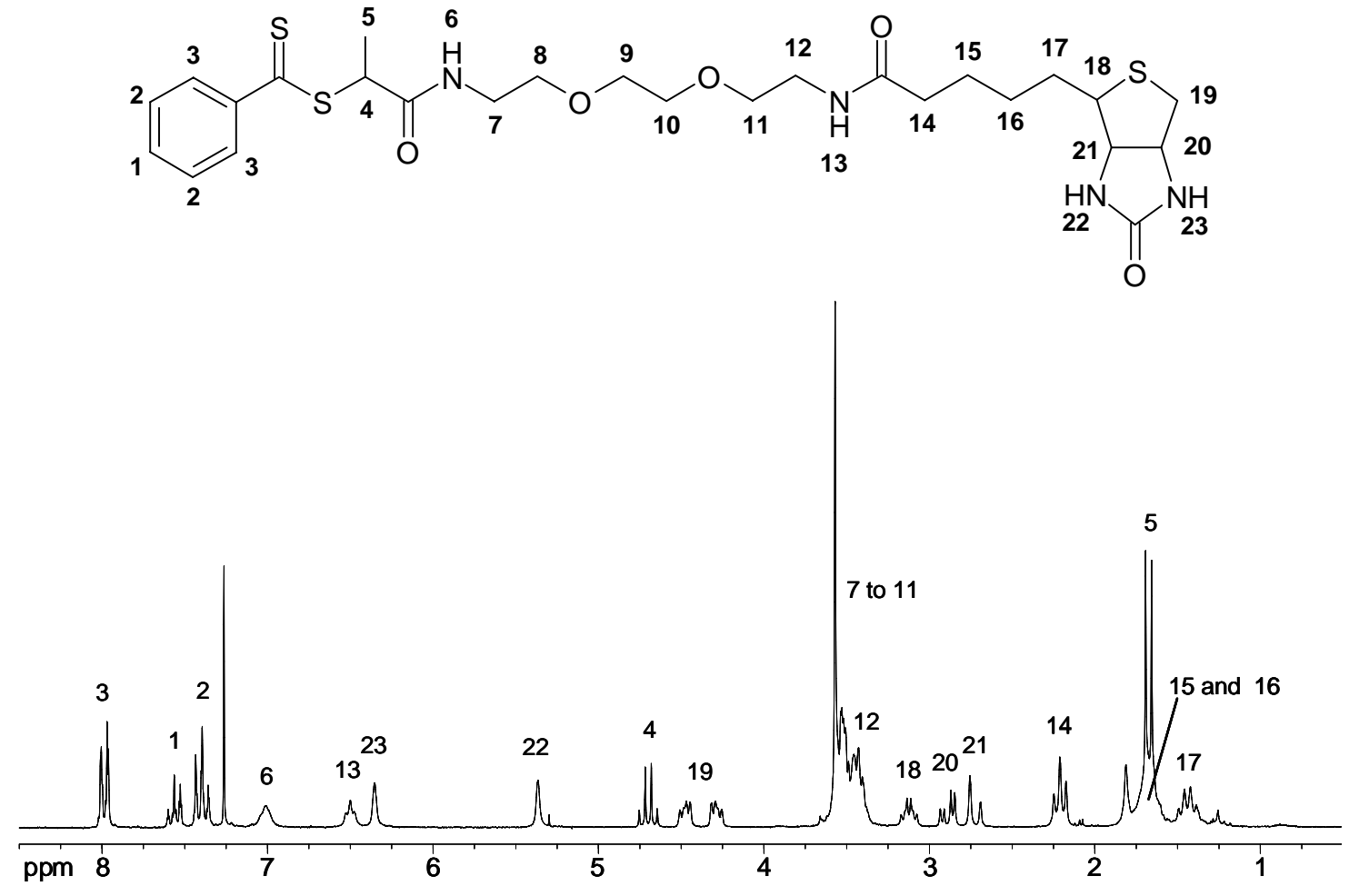

Figure 7: ${ }^{1} \mathrm{H}$ NMR spectrum of RAFT agent $3 \mathrm{c}$ in $\mathrm{CDCl}_{3}$.

${ }^{13} \mathrm{C}$ NMR 50MHz ( $\mathrm{CDCl}_{3}, \mathrm{RT}$, ppm) (see structure below for carbon assignment): $16.43\left(\mathrm{C}_{7}\right)$; $25.65\left(\mathrm{C}_{19}\right)$; 28.13 and $28.29\left(\mathrm{C}_{17}\right.$ and $\left.\mathrm{C}_{18}\right)$; 36.04, 39.12, 39.59, $40.50\left(\mathrm{C}_{9}, \mathrm{C}_{14}, \mathrm{C}_{16}, \mathrm{C}_{21}\right)$; $48.94\left(\mathrm{C}_{6}\right)$; $55.69\left(\mathrm{C}_{20}\right)$; 60.26 and $61.81\left(\mathrm{C}_{22}\right.$ and $\left.\mathrm{C}_{23}\right)$; $67.09\left(\mathrm{C}_{10}\right)$; 69.65, 69.97 and 70.22 $\left(\mathrm{C}_{11}, \mathrm{C}_{12}\right.$ and $\left.\mathrm{C}_{13}\right) ; 127.01\left(\mathrm{C}_{3}\right) ; 128.51\left(\mathrm{C}_{2}\right) ; 133.03\left(\mathrm{C}_{1}\right) ; 144.29\left(\mathrm{C}_{4}\right) ; 162.03\left(\mathrm{C}_{24}\right) ; 170.64$ $\left(\mathrm{C}_{8}\right) ; 173.38\left(\mathrm{C}_{15}\right) ; 227.41\left(\mathrm{C}_{5}\right)$. 

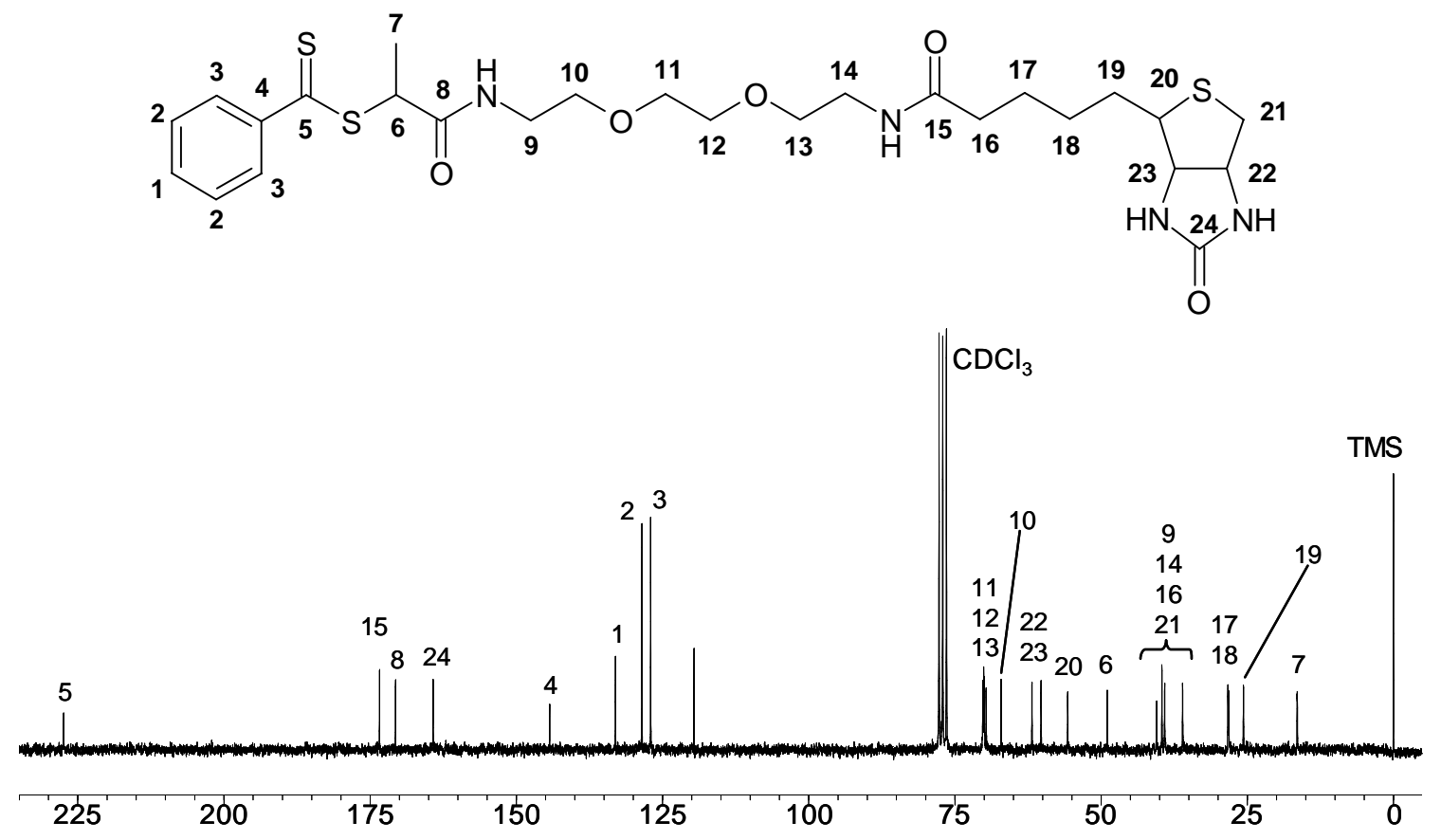

Figure 8: ${ }^{13} \mathrm{C}$ NMR spectrum of RAFT agent $3 \mathrm{c}$ in $\mathrm{CDCl}_{3}$.

Fast Atom Bombardment (FAB) mass spectrometry: characteristic ion $\left[\mathrm{M}+\mathrm{H}^{+}\right]$: $\mathrm{C}_{26} \mathrm{H}_{39} \mathrm{~N}_{4} \mathrm{O}_{5} \mathrm{~S}_{3}$; calculated 583.2083; found 583.2050.

Anal. Calcd. for $\mathrm{C}_{26} \mathrm{H}_{38} \mathrm{~N}_{4} \mathrm{O}_{5} \mathrm{~S}_{3}$ : C, 53.58; H, 6.57; N, 9.61; S, 16.50. Found: C, 53.01; H, 6.68; N, 9.29; S, 16.40

Synthesis of succinimido-4-[[2-phenyl-1-thioxo]thio]-4-cyanopentanoate (precursor RAFT agent 4).

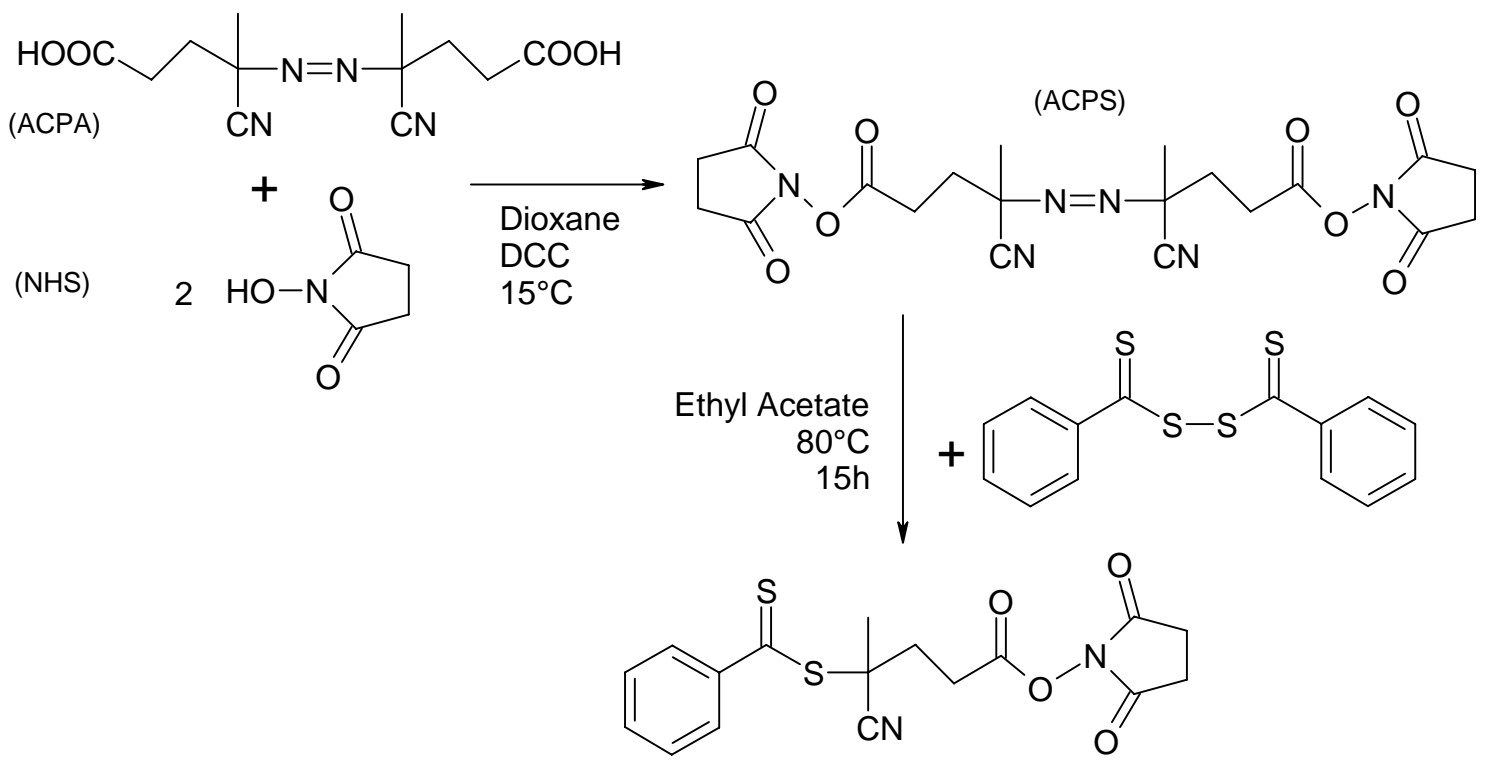

Scheme 1: Synthetic scheme for succinimido-4-[[2-phenyl-1-thioxo]thio]-4-cyanopentanoate

A solution of 4,4'-azobis-[4-cyanopentanoic acid] (ACPA, $2.42 \mathrm{~g}, 8.6 \mathrm{mmol}$ ) and $\mathrm{N}$ Hydroxysuccinimide (NHS, $2.98 \mathrm{~g}, 25.9 \mathrm{mmol}$ ) in dioxane (200 mL) was purged by nitrogen 
for 1 hour. $N, N^{\prime}$-Dicyclohexylcarbodiimide (DCC, 7.14g, $34.6 \mathrm{mmol}$ ) was slowly added at $15^{\circ} \mathrm{C}$ under vigorous stirring. After 15 hours at room temperature, the reaction mixture was filtrated. The azo-compound obtained (ACPS) was purified by two precipitations in cyclohexane and in diethyl ether at $4^{\circ} \mathrm{C}$. Bis(thiocarbonyl) disulfide was synthesized according to the literature procedure ${ }^{4}$. A solution of ACPS (945 mg, $1.99 \mathrm{mmol}$ ) and bis(thiocarbonyl) disulfide (407 mg, $1.33 \mathrm{mmol}$ ) in ethyl acetate $(60 \mathrm{~mL})$ was heated at $80^{\circ} \mathrm{C}$ for 16 hours. The reaction mixture was filtrated, and after drying under vacuum, a crystallized orange product was obtained.

${ }^{1} \mathrm{H}$ NMR $200 \mathrm{MHz}\left(\mathrm{CDCl}_{3}, \mathrm{RT}\right.$, ppm) (see structure below for proton assignment): 1.96 (s, $\mathrm{H}_{4}$ ); 2.59 (m, $\mathrm{H}_{5}$ ); 2.74 (m, $\mathrm{H}_{5}$ ); 2.86 (s, $\mathrm{H}_{7}$ ); 3.00 (m, $\mathrm{H}_{6}$ ); 7.41 (dd, $\mathrm{H}_{2}$ ); 7.58 (dd, $\mathrm{H}_{1}$ ); 7.91 (d, $\left.\mathrm{H}_{3}\right)$.

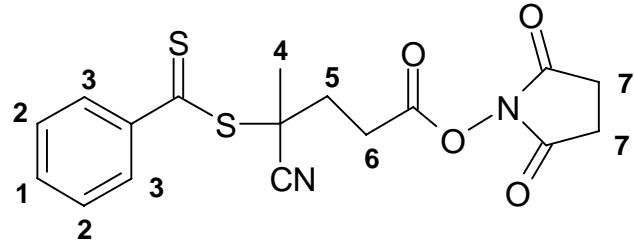

\section{References}

D'Agosto, F.; Charreyre, M.-T.; Veron, L.; Llauro, M.-F.; Pichot, C.

Macromol. Chem. Phys. 2001, 202, 1689-1699.

D'Agosto, F.; Hughes, R.; Charreyre, M.-T.; Pichot, C.; Gilbert, R. G. Macromolecules 2003, 36, 621-629.

Badey, B.; Boullanger, P.; Domard, A.; Cros, P.; Delair, T.; Pichot, C. Macromol. Chem. Phys. 1996, 197, 3711-3728.

Sprong, E.; De Wet-Roos, D.; Tonge, M. P.; Sanderson, R. D.

Journal of Polymer Science Part A: Polymer Chemistry 2003, 41, 223-235. 